\title{
EXPLORING THE PROBLEMS FACED BY NON-ENGLISH LECTURERS OF UNIVERSITAS RIAU KEPULAUAN ON USING MACHINE TRANSLATION ON ACADEMIC PURPOSE
}

\author{
Erwin Ashari \& Shalehoddin \\ University of Riau kepulauan, Batam \\ Corresponding: erwinashariharianja83@gmail.com
}

\begin{abstract}
The aim of this study was to measure the difficulties on using machine translation on writing article. It was a qualitative research by using descriptive analysis. Questionnaire was used to collect data; the questionnaire was shared to all lecturers of Riau Kepulauan University, as the respondents. Results show, respondents stated that Machine Translation (Google Translate) has important role on writing their scientific text.. respondents translate sentence, it purpose to confirm the structure and grammar of the sentence. Respondents used to translate from English to Indonesian to understand, and vice versa, to justify and confirm the difficult words in using Machine Translation. The words and phrases can be detected, but sometimes the meaning is different in the scientific field. So, they thought that it needs it to be developed. The effect caused by respondents after using Machine Translate is that respondents become lazy to open a dictionary. This feeling of laziness arises because the process of using Machine Translation is practice and efficient. The benefits of using it are can reduce the time on translating and understanding text English into Indonesian and vice versa. Second, it make easy to understand English text or Indonesian text because the user got a descriptions about the text of definition. Third, it can enrich the vocabularies of the user while using it, because while using it, user are served by several options depends on the discipline.
\end{abstract}

Keywords; Idiom, Principle of Translation, Google Translation

\section{INTRODUCTION}

It is a must for every lecturer to do research and then research findings are dessiminated to the world as the new information, science, and knowledge through proceeding, book, handbook, journal or seminar. This is a one of the "Tri Darma Perguruan Tinggi", namely Research. The findings of research are published in national or internation journal. A good research is a research that has a good state of art and solving gap of a research. It means that a researcher must reads a lot research findings that related to his/her research, so there is no repetation of the reseach on the same filed or case, then make them becomes as his/her reserach references.

If only one the number of language in the world, it does not need a translation study. But in the fact, the number of language in the word is similar to 
the number of the culture. every language has own writing, sound, and sturucture/grammar system, these differences differ one language to others. So, to communicate the among difference culture needs a tool, that is translation., and translation itself is rendering the meaning of a text into another language in the way that the author intended the text (Newmark, 1988). It shows that translation "bridge" communation of different language users.he expectation of reading and understanding reseach findings becomes as a phenomena for some lecturers, especially in University of Riau Kepulauan, because the reseach language is not always written in Bahasa Indonesia, it is written in English or other international languages, either local, national or international journals. Beside it, not all of them can understand English, spoken nor written.

So the only solution is by using machine translation as a tool of translaion. Eventhouh the accuracy of machine translation sometimes lead to the misperception. (CRC, 2014) says machine translation is software or online applications that automatically translate written material from one language to another without the involvement of a human translator or reviewer.

According to Hutchins in (Ali, 2016) Based on the common claim that a Machine translation is a substitute to the human translators, which is not true because Machine Translation systems are often measured to be inadequate and accused of not living up to the intention they made for. However, if machine translation is consider as translation tools or communication aids rather than as a replacement for an individual translator, it will be discovered that they are significant and often are widely underestimated.

The need for machine translation can be briefly stated into following points briefly: too much to be translated, boring for human translators, major requirement that terminology used consistently, increase speed and throughput, top quality translation not always needed, reduced cost (Ruchika A. Sinhal, 2014). Meaning is represented by expression or text and expression represents meaning. It means, to express meaning, people use texts or expression. Every text has own meaning that is influenced by the context (Ashari, 2015). In Indonesian context, "you" should be translated into "ibu". Meanwhile in sentence 2) this is Rina's book. In machine translation does not use apostrophe to show possessive pronoun 
and article cannot be used before the proper noun such as: name of person and place. As the researcher observed most of University of Riau Kepulaun lecturers used the machine translation when they were translating article but most of them still faced many problems in translating. According to the explanation above, the researcher thinks it is imortant to do research entitled "Exploring the Problems Faced by Non-English Lecturers Of Universitas Riau Kepulauan On Using Machine Translation On Academic Purpose.

Here are some definitions about machine translation (MT) by some experts. (Kunchukuttan, 2006) says Machine translation is automatic conversion of text/speech from one natural language to another. Meanwhile, (CRC, 2014) said Machine translation is software or online applications that automatically translate written material from one language to another without the involvement of a human translator or reviewer. Translator must maintain structure of original meaning, keeping ideas and facts accurate. Translator also must transmit cultural references, including slang, and other expressions that do not translate literally.

(Shuttleworth \& Cowie, 2014) said Machine Translation (MT) or Automatic Translation is a term used to refer to translation which is performed wholly or partly by computer. As implied by this definition, such translation may be carried out with or without human intervention; however, if there is a considerable level of intervention, or if computer applications are simply used as "translation tools", then it is more common to talk of MACHINE-AIDED TRANSLATION, although the boundary between these two approaches is not always completely clear-cut.

In addition (Hutchins, 2003) said machine translation (MT) refers to computerized systems responsible for the production of translations with or without human assistance. Also (Chan, 2015) Machine translation (MT) is a subfield of computational linguistics (CL) or natural language processing (NLP) that investigates the use of software to translate text or speech from one natural language to another.

Based on the definitions of machine translation from the experts above, it can be concluded that machine translation is the production of text in one natural language from that in another by application of computer. 
According to Vauquois in (Chan, 2015) MT approaches can be categorized by the depth of intermediary representations which are used in the translation process: direct, transfer and interlingua, which are often depicted by the Vauquois' Triangle (Vauquois 1968: 254-260).

In this triangle, shown in Figure 2.2, transfer approach is subcategorized to syntactic transfer approach

\section{Direct}

In the direct approach, the target language sentence is translated directly from the source word sequence, possibly with analysis in certain individual linguistic phenomena. No general syntactic or semantic analysis is conducted and the most (even only) important resource for this approach is a dictionary. That is the reason why it is also called dictionary-based approach.

\section{Transfer}

In the transfer approach, the intermediary structures are defined for both source and target languages. The translation process is divided into three phases: analysis from the source language sentence to the source intermediary structure; transfer from the source intermediary structure to the target intermediary structure; generation from the target intermediary structure to the target sentence.

Independent analysis means the characteristics of the target language are not considered in the analysis phrase, which will lead to a source intermediary structure which can be used for MT into various target languages. Independent generation means the characteristics of source language are not considered in the generation phrase, that is to say, the target intermediary structure can be used for MT from various source languages.

In the syntactic transfer approach the transfer process mainly occurs at the syntactic level. Thus the system may have components of morphological analysis, syntactic analysis, syntactic transfer, syntactic generation and morphological generation. In the semantic transfer approach the transfer process mainly occurs at the semantic level.

\section{Interlingua}

In the interlingua approach, a universal representation is defined for all the source and target languages. The translation process only contains two phrases: 
analysis and generation. The interlingua approach is regarded as an appropriate method for multilingual MT because it dramatically reduces the number of components compared with what is needed in direct approach or transfer approach. An interlingual can be a structured representation such as a logic expression, a semantic network, or a knowledge representation and so on, or an artificial or natural language representation.

An interlingua is also called a pivot language, a metalanguage or a bridge language. Practices in some large-scale projects showed that an interlingua approach using a human-defined presentation may encounter uncontrollable complexity when many languages are involved. Recent web-based translation services such as Google Translate, Bing Translator, etc. usually adopt English as a pivot language to support MT between tens of other languages.

\section{Problem of Machine Translation}

Beside machine translation can shorten time and make easier translator in translation, it does not mean that machine translation has not problems. So in this study, the researcher will explain about some problems used machine translation system. As (Arnold, Balkan, Humphreys, Meijer, \& Sadler, 1994) said there are three problems used machine translation:

\section{Ambiguity}

When a word has more than one meaning, it is said to be lexically ambiguous. When a phrase or sentence can have more than one structure it is said to be structurally ambiguous. Ambiguity is a pervasive phenomenon in human languages. It is very hard to find words that are not at least two ways ambiguous, and sentences which are (out of context) several ways ambiguous are the rule, not the exception.

\section{Lexical and Mismatches}

Some of these problems are to do with lexical differences between languages - differences in the ways in which languages seem to classify the world, what concepts they choose to express by single words, and which they choose not to lexicalize. We will look at some of these directly. Other problems arise because different languages use different structures for the same purpose, and the same structure for different purposes. In either case, the result is that we 
have to complicate the translation process. In this section we will look at some representative examples.

\section{Multi word units : Idiom and collocation}

Idioms are expressions whose meanings cannot be completely understood from the meanings of the component parts. In many cases, a natural translation for an idiom will be a single word. One problem with sentences which contain idioms is that they are typically ambiguous in the sense that either a literal or idiomatic interpretation is generally possible. This problem does not arise with all idioms. Some are completely frozen forms whose parts always appear in the same form and in the same order.

\section{RESEARCH METHODOLOGY}

In this study, the researcher uses descriptive qualitative research since it emphasizes on describing the phenomena of the use of language in its context by interpreting the data. This study mainly used the descriptive qualitative method. (Prior, 2008) said that Qualitative methodology is a composite of philosophy, concepts, data-gathering procedures, and statistical methods that provides perhaps the most thoroughly elaborated basis for the systematic examination of human subjectivity.

In addition (Creswell, 2014) said that qualitative methods rely on text and image data, have unique steps in data analysis, and draw on diverse designs. Writing a methods section for a proposal for qualitative research partly requires educating readers as to the intent of qualitative research, mentioning specific designs, carefully reflecting on the role the researcher plays in the study, drawing from an ever-expanding list of types of data sources, using specific protocols for recording data, analyzing the information through multiple steps of analysis, and mentioning approaches for documenting the accuracy — or validity — of the data collected.

(Keegan, 2009) said that qualitative research is less easy to define. It explores questions such as what, why and how, rather than how many or how 
much; it is primarily concerned with meaning rather than measuring. Although we may refer to the outcomes of qualitative research as data, they are not data in the sense of being facts or statistics. They refer to behavior, thoughts, opinions, meaning and the like. Qualitative researchers need to be highly skilled and competent in all stages of the research process In this study, the researcher will conduct the research at University of Riau Kepulauan which is located on J1. Batu Aji baru No. 99 Batam. University of Riau Kepulauan has some of faculties; FKIP (Faculty of Teacher Training and Education), faculty of economy, faculty of law, faculty of social poitics and faculty of engeneering. This research will be hold on April 2019. The subjects of this study are all lecturers of University o Riau kepulauan except English departmen lecturers with amount; 154 lecturers. . .

In this study, the researcher apply questionnaire as a tool to gather data or information. According (Prior, 2008) a questionnaire is seeking data about service delivery in a nonprofit setting may include space for open-ended comments where respondents can indicate why they responded in certain ways or provide details not otherwise captured by closed response items. The questionnaire was conducted, particularly to reach the objectives of this present study. The questionnaire which cover five specific question that consist 20 items about frequency on using machine translation; the usage on using machine translation; the difficulty on using machine translation; the weakness on using machine translation; and the solution on using machine translation.

Techniques of collection data are the most important step in this study, because the main purpose of this study is to get the relevant data. Without knowing the techniques of collecting data, the researcher will not get the appropriate data. According to (Creswell, 2014) The data collection steps include setting the boundaries for the study, collecting information through unstructured or semi structured observations and interviews, documents, and visual materials, as well as establishing the protocol for recording information. According to Bodgan in (Sugiono, 2010) data analysis is the process of systematically searching and arranging the interview transcripts, field notes, and other materials that you accumulate to increase your own understanding of them and to enable you to present what you have discovered to others. Also, Stainback in (Sugiono, 2010) 
said that data analysis is critical to the qualitative research process. It is to recognition, study, and understanding of interrelationship and concept in your data that hypotheses and assertions can be developed and evaluated.

\section{RESULT AND FINDING}

After collecting the questionaire, the researchers did clasification based on the qestionaire questions. Beside it, the researchers did write the notes, then the results determnined based on the the respondents' answers. To make it clear, it can be seen as follows;

1. Have you ever used Machine Translation (Google Translate) on writing and understanding scientific text?

Based on the result, it shows that the respondents used Machine Translation (Google Translate) on writing and understanding scientific text. But, a several respondents did not used Machine Translation (Google Translate) on writing and understanding scientific text. When the answered was "no", so the next column must be empty, but after confirming the meaning of the questionaire, they changed their anwers, because the thought it was only Indonesian to English. The dominant answers, it shows that google translate is familiar to respondents.

2. Does Machine Translation (Google Translate) has important role on writing your scientific text?

Based on the result, it shows the respondents stated that Machine Translation (Google Translate) has important role on writing their scientific text, and a several respondents stated Machine Translation (Google Translate) has not important role on writing their scientific text but this answer was categorized as unhonest answer. And then they were explained about the purpose of the questionaires, then they had changed their answers.

3. What percentage of the role of Machine Translation (Google Translate) on writing your scientific text?

Based on the result, the respondents used Machine Translation (Google Translate) in $41-60 \%$ in average. It means that they have frontier 
knowledge in English. In another words they just want to justify and confirm the difficult words in using Machine Translation. But some of them answered orally that they needed every writing scientific article, it means that actually the used it in high percentage of use. This condition shows that the google translation was their need on writing scientific article.

4. In translating using Machine Translation (Google Translate), do you translate by word, phrase, sentence or paragraph?

Based on the result, dominantly the respondents translate sentence, it purpose to confirm the structure and grammar of the sentence, it means that the respondents has English knowledge. Respondents translate paragraph in order to get and understand the main idea of the text, it is used to translate English to Indonesian. Meanwhile, a several respondents translate words to discover difficult words and translate phrase to get the idioms definition.

5. According to you, which is easier, translate the English text into Indonesian or vice versa by using machine translation?

Based on the results, respondents use both. respondents use translate from English to Indonesian to understand, and vice versa. This answer related to the previous questions. It means that it is important for them.

6. Is the translation result from machine translation easy to understand?

Based on the results of respondents exposure, the translation results from Machine Translation are easily understood, some respondents also consider it very difficult to understand because because the grammar of Google Machine is not standard so it still needs to be improved. Beside it that there were some options on translation it. It make they felt difficulties.

7. Does machine translation help you to find and understand other scientific articles?

Based on the results, the most of respondents is helped by the existence of Machine Translation in finding and understanding other scientific text. A several respondents stated that Machine Translation isn't helping them, 
they prefer to used other applications, namely Merriam Webster and also difficult to understand when translating English text into Indonesian.

8. Are all the words and phrases that you are looking for can be detected by Machine Translation?

Based on the result, the most of respondents stated that the words and phrases can be detected, but sometimes the meaning is different in the scientific field. Meanwhile a several respondents stated it can't be detected because some majors are not detected. So, they thought that it needs it to develop.

9. What are the obstacles that you usually face when using Machine Translation when writing scientific articles?

Based on the result of the respondents, the obstacles is the Machine Translation language is not scientific, the language is ambiguous, the language is not detected, a different meaning and the grammar is wrong. It can be conclude that the most of obstacles is the language of Machine Translation not good.

10. Are the results of the translation of scientific text using Machine Translation in accordance with the principles of translation?

Based on the result, dominantly the respondents stated the result of the translation is not accordance with the pronciple of translation because there is still mistake while translating, the mistake can be caused by the idioms.

11. What steps do you take if the translation of the machine is not considered appropriate?

Based on the result, steps taken by the respondent when the results of Machine Translation were not correct yet, the respondents used an English-Indonesian dictionary and a merriam webster dictionary. Then, some respondents ask their experts or ask colleagues. While other respondents use other better applications.

12. According to you, what is the effect that arises if you use Machine Translation intensively? 
Based on the result, the effect caused by respondents after using Machine Translate is that respondents become lazy to open a dictionary. This feeling of laziness arises because the process of using Machine Translation is practice and efficient. However, several other respondents stated that Machine Translation helps them translate, provide easy access and save time.

13. What should you do after knowing the effects of using machine translation?

Based on the previous result, it shows that it is not good to use Machine Translation for a long time or make Machine Translation as the only recources. So, they suggested to avoid of using it intensively, and also they suggested to used printed dictionary or ask another person who knows English well.

14. What are the benefits that you get from using machine translation?

The benefits of using it are can reduce the time on translating and understanding text English into Indonesian and vice versa. Second, it make easy to understand English text or Indonesian text because the user got a descriptions about the text of definition. Third, it can enrich the vocabularies of the user while using it, because while using it, user are served by several options depends on the dicipline.

\section{REFERENCES}

Ali, A. (2016). Exploring The Problems Of Machine Translation From Arabic Into Englishlanguage Facedby SaudiUniversity Student Of Translation At The Faculty-- Of Arts,Jazan UniversitySaudi Arabia. IOSR Journal of Humanities and Social Science, 21(4), 55-66. https://doi.org/10.9790/08372104025566

Amberg, J. S., \& Vause, D. J. (2006). Introduction: What is language? Cambridge: Cambridge University Press.

Arnold, D., Balkan, L., Humphreys, R. L., Meijer, S., \& Sadler, L. (1994). Machine Translation: An Introductory Guide. Machine Translation. london: NCC Blackwell Ltd. Retrieved from http://www.essex.ac.uk/linguistics/external/clmt/MTbook/HTML/book.html 
Arrahman. (2005). An analysis of english-indonesian translation. FACULTY OF ADAB AND HUMANITIES SYARIF HIDAYATULLAH STATE ISLAMIC UNIVERSITY JAKARTA 2005.

Ashari, E. (2015). Patterns of Coding in Conversation Texts of the English Zone. Anglo-Saxon, 6(8), 39-46.

Basnet, S. (2014). Translation. London and New york: Routledge, Taylor and Francis Group.

Bassnett, S. (2005). Translation studies. Vasa (third edit). London and New york: Routledge, Taylor and Francis Group. https://doi.org/10.1017/CBO9781107415324.004

Chan, S. (2015). The Routledge encyclopedia of translation technology. (S. Chan, Ed.) (first, p. 757). London and New york: Routledge, Taylor and Francis Group. https://doi.org/10.4324/9781315749129.ch3

CRC, M. M. D. R. R. (2014). Machine translation. English, 26.

Creswell, J. W. (2014). Research Design: Qualitative, Quantitative, and Mixed Methods Approaches. Research design Qualitative quantitative and mixed methods approaches (fourth). sage. https://doi.org/10.1007/s13398-0140173-7.2

House, J. (2015). Translation quality assessment: Past and present (first). London and New york: Routledge, Taylor and Francis Group.

Hutchins, J. (2003). Machine Translation: General Overview. The Oxford Handbook of Computational Linguistics. Retrieved from http://www.hutchinsweb.me.uk/Mitkov-2003.pdf

Keegan, S. (2009). Qualitative Research - Good Decision Making Through Understanding People, Cultures and Markets. (David barr and robin J birn, Ed.), Zhurnal Eksperimental'noi $i$ Teoreticheskoi Fiziki. Lomdon \& Philadelphia: Marker research in practiceseries. https://doi.org/10.2501/S1470785309201247

Krippendorff, K. (2004). Content Analysis: An Introduction to Its Methodology. Education (second, Vol. 79). london: sage. https://doi.org/10.2307/2288384

Kunchukuttan, A. (2006). Machine translation. English, 53. Retrieved from anoopk@cse.iitb.ac.in www.cse.iitb.ac.in/ anoopk

Mughazy, M. (2016). The Georgetown guide to Arabic-English translation. Washington DC: Mustafa mughazy. (2016). The Georgetown Guide to Arabic-English Translation. Washington DC. 
Munday, J. (2016). Introducing translation studies Theories and applications. London: Routledgf (fourth). London and New york: Routledge, Taylor and Francis Group. Retrieved from https://scholar.google.co.uk/scholar?start=340\&q=translation+studies\&hl=en \&as_sdt=0,5\#1

Newmark, P. (1988). A Textbook of Translation. Text. Shanghai: SHANGHAI FOREIGN LANGUAGE EDUCATION PRESS.

Prior, L. (2008). Document analysis. ( lisa m Given, Ed.), The Sage Encyclopedia of Qualitative Research Methods (1st \&2nd ed.). london: a sage reference publication. https://doi.org/10.4135/9781412963909

Ruchika A. Sinhal, K. O. G. (2014). Machine Translation Approaches and Design Aspects. Http://www.iosrjournals.org/iosr-Jce, 16(1), 22-25. Retrieved from http://www.iosrjournals.org/iosr-jce/papers/Vol16-issue1/Version2/D016122225.pdf

Shuttleworth, M., \& Cowie, M. (2014). Dictionary of Translation Studies. London and New york: Routledge, Taylor and Francis Group.

Sugiono. (2010). metode penelitian pendidikan (9th ed.). bandung: alfabeta. 